\title{
Uma Proposta de Framework para Apoiar o Planejamento de Trabalhos em Grupo Baseados em Project Based Learning
}

\author{
Edmar Welington Oliveira ${ }^{1}$, Marcos Roberto S. Borges ${ }^{2}$, Welington Veiga ${ }^{3}$ \\ ${ }^{1,3}$ Departamento de Ciência da Computação - Universidade Federal de Juiz de Fora \\ (UFJF) - Juiz de Fora, MG - Brasil \\ ${ }^{2}$ Departamento de Ciência da Computação - Universidade Federal do Rio de Janeiro \\ (UFRJ) - Rio de Janeiro, RJ - Brasil \\ \{edmar.oliveira, welington.veiga\}@ice.ufjf.br, mborges@nce.ufrj.br
}

\begin{abstract}
This research is focused on the difficulties inherent to the process of planning collaborative group work scenarios. It presents a study that shows some issues and inefficiencies associated with the process. The results show that it is crucial to provide educators with proper and useful guidance. In this sense, it is proposed a framework to support educators to design collaborative learning scenarios based on PBL principles.
\end{abstract}

Resumo. Este trabalho está focado nas dificuldades inerentes ao processo de planejamento de cenários colaborativos. Apresenta-se um estudo que expõe os problemas e ineficiências associadas ao processo. Os resultados indicam ser essencial prover educadores com adequada orientação. Portanto, é proposto um framework para auxiliá-los no processo de planejamento de trabalhos em grupo baseados no modelo instrucional Project Based Learning.

\section{Introdução}

Processos instrucionais nem sempre são efetivos [Kimble et al., 2008]. Portanto, os objetivos inerentes à aquisição/desenvolvimento de habilidades e conhecimentos nem sempre são alcançados. Há um crescente interesse no uso da aprendizagem colaborativa (AC) de modo a se prover cenários que oportunizem aos alunos alcançar esses objetivos [Isotani et al., 2013]. Contudo, o simples fato de se ter estudantes trabalhando juntos não garante uma aprendizagem efetiva, ainda que recursos colaborativos lhe sejam providos [Weinberger et al., 2009]. Espera-se dos educadores a capacidade de criar cenários que mantenham alunos ativamente envolvidos na construção do próprio conhecimento; que, sobretudo, sejam adaptados a eles. A chance de se obter uma aprendizagem significativa e duradoura diminui sensivelmente quando não são apropriadamente planejados [Isotani et al., 2010]. De fato, planejamento inadequado é uma das principais razões de insucesso da aprendizagem em grupo [Dillenbourg, 2002].

Projetar cenários de AC é complexo, pois envolve inúmeros requisitos, variáveis e restrições [King, 2007]. Consequentemente, são indevidamente [Barkley et al., 2014] e ineficientemente estruturados [Höver \& Muhlhauser, 2014] - dificultando ou impedindo que alunos alcancem objetivos de aprendizagem. Trabalho em grupo, sobretudo quando o foco é aprendizado, requer planejamento cuidadoso [Dillenbourg, 2002]. Embora seja uma prática coletiva, é essencial ponderar sobre as necessidades individuais dos alunos. Soma-se à complexidade, transformar essas necessidades, e as intenções dos educadores, 
VI Congresso Brasileiro de Informática na Educação (CBIE 2017)

Anais do XXVIII Simpósio Brasileiro de Informática na Educação (SBIE 2017)

em parâmetros/variáveis que estruturem o trabalho. Esse é um processo particularmente desafiador para, mas não limitado a, educadores iniciantes - que, em geral, não possuem conhecimento e experiência necessários para planejar, adequadamente, cenários de AC [Isotani et al., 2013].

O problema de pesquisa refere-se à falta de planejamento adequado de trabalhos em grupo. Portanto, propôs-se analisar as dificuldades inerentes ao processo. O objetivo foi investigar e obter informações acerca da forma como docentes, do ensino superior da área de computação, realizam o planejamento de trabalhos em grupo ao lecionarem suas disciplinas na graduação presencial. Identificados os problemas/ineficiências, o enfoque de solução baseia-se na proposição de um framework apto a guiar/orientar os docentes no processo. As contribuições consistem em (a) discutir os problemas identificados e (b) propor uma infraestrutura capaz de expor os educadores a parâmetros de planejamento de modo que possam ser, efetiva e sistematicamente, utilizados. Nesse sentido, realizouse um estudo exploratório com o propósito de obter informações quanto ao processo de planejamento realizado pelos docentes. O método qualitativo-quantitativo, aplicado ante uma amostragem não probabilística e intencional, foi utilizado. Os dados foram obtidos através de entrevistas. Análise de conteúdo e estatística foram usadas para examiná-los. As informações obtidas fundamentaram a proposição do framework. Particularmente, $o$ intuito da pesquisa é oportunizar o planejamento de cenários de $\mathrm{AC}$ baseados no modelo instrucional Project Based Learning (PBL). O interesse por PBL está no fato do mesmo possibilitar tanto aquisição de conhecimento quanto desenvolvimento de indispensáveis habilidades sociais e profissionais. Assim, preparando os aprendizes para situações que vivenciarão em ambientes reais de trabalho.

O trabalho está organizado conforme a seguir. A seção 2 apresenta os resultados obtidos através das entrevistas. A seção 3 apresenta a análise dos dados e discute alguns dos problemas observados. O framework proposto é descrito na seção 4. Considerações finais, limitações da pesquisa e trabalhos futuros são apresentados na seção 5 .

\section{Planejamento de Trabalhos em Grupo: Um Panorama}

Para esta pesquisa, realizou-se um estudo exploratório. O objetivo foi investigar como professores, do ensino superior presencial em computação, planejam trabalhos em grupo ao ministrarem suas disciplinas de graduação. Foram entrevistados 30 indivíduos do departamento de ciência da computação de uma Universidade Federal. Optou-se pela realização de entrevistas semiestruturadas. Seguiu-se um roteiro de perguntas abertas, as quais foram previamente estabelecidas. Na presença do entrevistado, foram verbalmente realizadas em ordem previamente definida. O uso desse tipo de entrevista possibilitaria a realização de perguntas complementares, a depender das circunstâncias momentâneas da mesma, buscando clareza nas respostas e/ou a coleta de mais informações. Ademais, permitiria a posterior comparação de respostas. Em média, duraram 1 hora. Todas foram gravadas, com autorização. A amostra era formada por 27 doutores e 3 mestres. Quanto à experiência em docência no ensino superior, tinha-se: 6 com menos de 5 anos; 7 entre 5 e $10 ; 5$ entre 11 e $15 ; 8$ entre 16 e 20; 2 entre 21 e $30 ; 2$ com mais de 31 .

Quanto ao uso da prática de trabalho em grupo, 22 a usam e 8, não. Destes 8, 5 já a usaram, mas abdicaram em razão da complexidade de se planejar os trabalhos. Os demais 3 nunca a utilizaram. Dado o objetivo da pesquisa, os resultados deste ponto em diante referem-se aos 22 professores que a utilizam. Apurou-se que nenhum docente usa 
VI Congresso Brasileiro de Informática na Educação (CBIE 2017)

Anais do XXVIII Simpósio Brasileiro de Informática na Educação (SBIE 2017)

modelos pedagógicos como suporte ao processo de planejamento. Todos se baseiam na própria experiência. Adotou-se a expressão "modelo pedagógico" como base comum às diferentes terminologias (p.ex., técnicas, pedagogias, teorias, padrões, etc.) empregadas no contexto de ensino/aprendizagem. Todos planejam/aplicam o mesmo trabalho para toda a turma. Logo, não se propõe trabalhos com características (complexidade, escopo, etc.) distintas, conforme as necessidades de aprendizado particulares dos alunos/grupos. Nenhum professor interfere na formação dos grupos.

Quanto à especificação das atividades, buscou-se averiguar (a) o quão granular são definidas, se: (b) a descrição é explícita ou implícita, (c) a duração é estimada, (d) os objetivos de aprendizagem são especificados, (e) relações de dependência entre elas são definidas, (f) suas execuções são monitoradas, (g) o nível de conhecimento "adequado" para sua execução é estipulado e (h) como são divididas nos grupos. Por granularidade, considerou-se o nível de divisibilidade da atividade - se pode ser decomposta em tarefas mais específicas. Nenhum especifica atividades com nível de granularidade alto (tarefas menores). Por descrição implícita, considerou-se aquela que está subentendida no texto do trabalho. Já a explícita se refere àquela claramente expressada. Dos 22, 16 descrevem as atividades explicitamente. Para o item "c", 21 não estimam duração. No item "d", 21 não definem objetivos de aprendizagem para tarefas. Para o item "e", considerou-se que relações de dependência entre tarefas são aquelas estabelecidas de forma que a execução de uma não pode ser finalizada sem a conclusão de outra(s). Logo, 21 não definem esse tipo de vinculação. No item "f", 16 não monitoraram a execução. No item "g", o nível de conhecimento adequado foi definido como sendo o intervalo entre o mínimo (exigido) e o máximo (permitido) para uma atividade ser realizada (evitando se apresentar ao aluno como algo excessivamente complexo/inviável ou fácil/desestimulante. Nenhum docente especifica atividades seguindo esse balizamento de conhecimento. No item "h", 21 não interferem na divisão das tarefas. Para 17, o principal critério usado pelos alunos para a divisão é a habilidade/conhecimento individual do aluno. Os alunos se responsabilizam, portanto, pelas atividades que lhe são mais fáceis.

Quanto à avaliação da aprendizagem, 19 adotam o produto (final e/ou parcial) do trabalho, e apresentações realizadas pelos alunos, como critérios. Dos 22, 20 avaliam os alunos individualmente, mas 14 não realizam uma análise a priori destes para aferir o aprendizado ao final do processo; 6 afirmaram fazê-la, mas informalmente (examinando desempenho em disciplinas anteriores, avaliações da própria disciplina, etc.). Dos 22, 20 definem a mesma nota para todos do grupo. Quanto às razões de alguns grupos falharem ao realizarem um trabalho, 15 alegaram a falta de organização dos alunos. Questionados sobre quem deveria ser responsabilizado nos casos de falha, 9 responderam os alunos; 2 , os docentes e 11, ambos. Os educadores por não projetarem adequadamente o trabalho e não monitorarem seu desenvolvimento; os alunos pela desorganização em relação à sua realização. Quanto ao trabalho coletivo, 13 disseram que os alunos são sabem trabalhar em grupo de forma efetiva, pois não sabem gerenciar (dividir atividades, interagir, etc.), adequadamente, a realização dos trabalhos. Para os 22, a principal motivação dos alunos para com trabalho em grupo é a execução do mesmo (cumprir os requisitos na disciplina - visando a aprovação) e não o aprendizado.

Para 21, a colaboração é importante para se promover a aprendizagem. Para 15, os alunos não possuem consciência do que é, de fato, colaborar. Mesmo assim, nenhum professor define diretrizes ou instruções para auxiliar os alunos a trabalharem juntos - a colaborarem. Para 15 docentes, realizar um planejamento detalhado pode fomentar um 
aprendizado efetivo - por propiciar orientar as ações dos alunos, incentivar interações e facilitar a avaliação e o monitoramento. Para 15, esse "detalhamento" é complexo, pois são muitos parâmetros a se considerar. Além disso, a maioria dos educadores não possui conhecimentos acerca de abordagens pedagógicas, que poderiam ajudá-los no processo. Para 7, não há complexidade; os educadores não o realizam por considerá-lo trabalhoso. Dos 22, 3 disseram realizar um planejamento detalhado; 7, não detalhado e 12, "médio". Para 20, seria benéfico dispor de infraestruturas (ferramenta, guia, etc.) para ajudar no processo de planejamento: monitoramento, controle de tarefas, análise da aprendizagem, etc. Dos 22, 13 não conhecem o modelo pedagógico PBL; 5 disseram ter "ouvido falar" (mas desconhecem seus conceitos); 4 conhecem, mas somente 1 já o utilizou.

\section{Análise dos Resultados}

As entrevistas salientaram vários problemas e ineficiências quanto ao processo de planejamento realizado pelos professores - principalmente com relação à orientação das ações dos alunos e a analise do aprendizado. Para a maioria dos docentes, os estudantes não sabem trabalhar em grupo de forma efetiva, não têm consciência do que é colaborar e estão, prioritariamente, empenhados tão somente na realização/entrega do trabalho. As consequências, diretas ou indiretas, englobam interações ineficazes/inexistentes, divisão indevida de tarefas/responsabilidades, má organização no desenvolvimento do trabalho e condutas desfavoráveis à aprendizagem (p.ex., aluno executar somente tarefas que lhe são mais "fáceis"). Além disso, a maioria crê que as falhas na realização do trabalho são decorrentes de más ações dos alunos - e quase a metade afirma que a responsabilidade é exclusiva dos mesmos. Apesar do exposto, o planejamento não é realizado de maneira a orientar os alunos. De fato, os professores não orientam a formação de grupos, a divisão de tarefas e responsabilidades, não definem diretrizes que fomentem a interação entre os alunos, não especificam detalhadamente as atividades (duração, relação de dependência, etc.) e não monitoram a execução das mesmas. É essencial que o trabalho seja planejado de maneira a orientar o aluno quanto à sua conduta, às atividades que deve executar e às interações com seus pares. Essa orientação faz-se necessária, pois os alunos (por vezes) não realizam atividades colaborativas adequadamente [Alharbi et al., 2014.]. Além disso, a "colaboração livre" (não estruturada) nem sempre produz resultados de aprendizagem satisfatórios [Alharbi et al., 2014; Dillenbourg, 2002; Barkley et al., 2014].

Quanto ao acompanhamento e análise do aprendizado, averiguou-se que não são especificados parâmetros que possibilitem ao docente realizá-los de forma precisa/exata - principalmente com relação ao aluno, individualmente. A maioria dos professores não realiza uma análise a priori dos estudantes (no que tange às suas necessidades/interesses de aprendizado, características, etc.). Além disso, o trabalho e as tarefas que o compõem não são estruturados de forma a atender às especificidades dos mesmos. Muitos utilizam o produto do trabalho para avaliar o aprendizado, mesmo não considerando que entregar o mesmo por si só (ainda que bem desenvolvido) seja garantia da aprendizagem. Outros, embora adotem apresentações, não especificam critérios, baseando-se no "feeling" ao se observar o aluno (sua capacidade de se expressar, responder, demonstrar conhecimento, etc.) durante as mesmas. Embora a maioria afirme avaliar os alunos individualmente, as notas não são individuais. Dado o exposto, somado ao fato de muitos não monitorarem o desenvolvimento do trabalho e/ou o processo de aprendizagem, é discutível a precisão e justeza dos professores quanto à percepção do aprendizado dos alunos. Em termos da AC, dois itens devem ser avaliados: o conhecimento do aluno quanto ao conteúdo e sua 
participação nos processos do grupo [Barkley et al., 2014]. Contudo, avaliar e monitorar o progresso individual em trabalhos em grupo é uma tarefa desafiadora [Fidalgo-Blanco et al., 2015] - justificando a proposição de infraestruturas que orientem os educadores.

\section{Framework para Planejamento de Trabalhos em Grupo com PBL}

Para assistir o processo de planejamento, optou-se por utilizar ambientes virtuais de aprendizagem (AVA). A proposta é viabilizar a integração de recursos educacionais em AVA como diretriz para planejamento de trabalho em grupo. A integração é direcionada no sentido de se possibilitar incorporar/adicionar soluções de terceiros; uma propriedade indispensável, dado o desafio destes ambientes em conseguir acompanhar as constantes inovações do domínio educacional. Logo, o uso de serviços web torna-se oportuno, pois estruturar recursos educacionais na forma de serviços permite que seu desenvolvimento (e evolução) ocorra alheio à plataforma e que eventuais correções se tornem disponíveis instantaneamente. Contudo, é quase inviável que alguma plataforma consiga incorporar todas as inovações/soluções, em virtude das especificidades e fragmentação das mesmas [Fragoso et al., 2014]. Uma alternativa reside na perspectiva de ecossistemas de software (ECO) [Santos, 2011], pois permite que diferentes organizações/instituições contribuam com soluções para a estruturação de ambientes de aprendizagem "ricos" em diversidade de serviços/soluções. Formalmente, um ECO "é uma interação de um conjunto de atores sobre uma plataforma tecnológica comum, resultando em um aglomerado de serviços de software. Cada ator é motivado por seus interesses, conectando-se aos demais e ao ECO como um todo por meio de relacionamentos simbióticos. A plataforma tecnológica está estruturada de forma a possibilitar o envolvimento e contribuição dos diferentes atores" [Manikas \& Hansen, 2013]. O framework proposto, portanto, visa transformar AVA em plataformas que sustentem um ecossistema - logo, permitindo que serviços voltados ao processo de planejamento sejam integrados.

\subsection{Metamodelo de Planejamento}

Integra o framework proposto uma estrutura conceitual de modelos em camadas - que conceitualizam o domínio de planejamento de trabalhos em grupo fundamentados em PBL. Tal estrutura inclui um metamodelo base (nível 1) - o qual descreve conceitos relacionados ao domínio de projeto - e metamodelos específicos (demais níveis). Todos estão inter-relacionados por meio de seus elementos conceituais comuns. O metamodelo de planejamento (nível 0) engloba toda essa estrutura conceitual. Tal organização busca “isolar", em camadas, os elementos conceituais característicos a cada domínio associado ao processo de planejamento, promovendo maior flexibilidade quanto ao uso e evolução da estrutura. O metamodelo base (figura 1) é embasado na especificação IMS-LD [IMS, 2003] e inclui conceitos intrínsecos ao domínio de projetos.

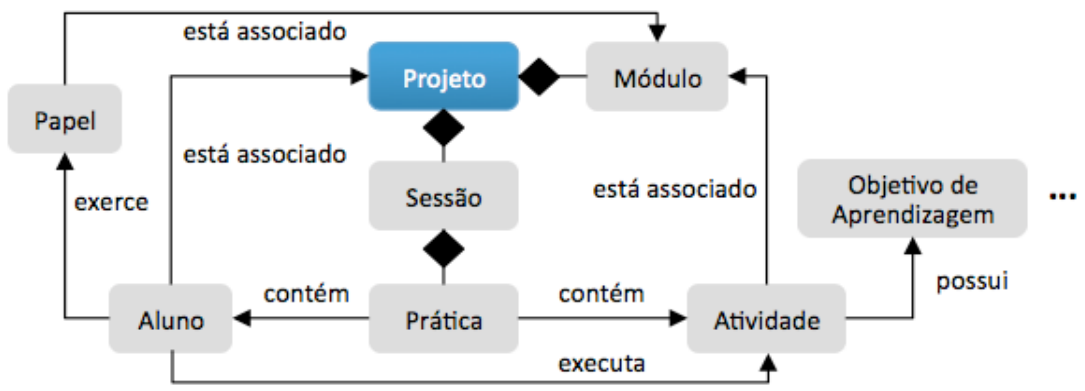

Figura 1. Fragmento do metamodelo de projeto (nível 1) 
VI Congresso Brasileiro de Informática na Educação (CBIE 2017)

Anais do XXVIII Simpósio Brasileiro de Informática na Educação (SBIE 2017)

Um projeto é composto de módulos (um exemplo é um projeto de software que envolve atividades nas áreas/módulos de programação, banco de dados, interface gráfica e redes). Um projeto ainda possui seções (etapas), onde práticas (execuções de tarefas) são realizadas. Uma atividade possui objetivos de aprendizagem e é executada por um aluno, exercendo um papel (ao qual estão associadas responsabilidades, competências e tarefas) específico no projeto. Atividades e papéis estão associados a um módulo. Além do metamodelo base, compõe o metamodelo de planejamento (nível 0) os metamodelos de: aprendizagem, colaboração, atividades, avaliação, grupos, alunos, suporte (conceitos que especificam ferramentas de apoio; por exemplo, de comunicação: chat, fórum, etc.), monitoramento e contingência (conceitos relativos à especificação de ações de resposta a ocorrências não previstas). Padrões e especificações (LOM [IEEE, 2002], ontologia de colaboração CONTO [Oliveira, 2009], etc.) respaldam a definição dos modelos. Outros têm sido estudados/avaliados de modo a apoiar a (re)estruturação dos mesmos.

\subsection{Arquitetura de Referência}

A arquitetura proposta neste trabalho é uma arquitetura de software de referência (ASR) [Muller, 2013] orientada a serviço web e independente de tecnologia/plataforma. Uma ASR é um modelo arquitetural que caracteriza funcionalidades computacionais de um domínio de aplicação específico, visando o entendimento deste e reuso da estrutura. A figura 2 apresenta a ASR proposta.

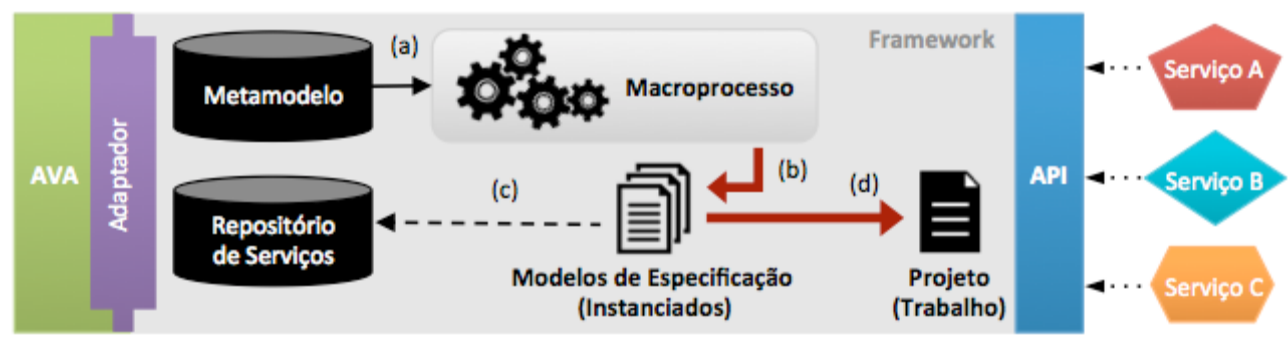

Figura 2. Arquitetura conceitual

A arquitetura inclui a API de integração - um conjunto de recursos para prover a comunicação entre serviços educacionais e a estrutura de ecossistema. Entre os recursos disponibilizados, está o que permite o registro e a recuperação de metadados de serviços educacionais no repositório de serviços. Recursos para segurança dos dados, controle de acesso, etc. também são definidos. Integra a arquitetura, o framework para planejamento de trabalhos em grupo embasados em PBL. Compõe este componente: o metamodelo de planejamento, o repositório de serviços educacionais, o macroprocesso de planejamento, os modelos de especificação e o projeto (ambos, produtos do planejamento). A conexão entre o framework e o AVA é realizada através do adaptador - uma camada de software na forma de plugin, extensão ou alteração no AVA. Sua função é traduzir as chamadas à API, que são independentes das plataformas AVA, para as especificidades destas. AVA e adaptador também integram a arquitetura. Compõe a estrutura de ecossistema a API, o framework e o adaptador. Ao implementar essa estrutura, o AVA se transforma em uma plataforma de ecossistema, capaz de sustentá-lo. O repositório de serviços é um canal de distribuição de serviços educacionais, similar a uma loja de aplicativos (como o Google Play) - que não contém os serviços em si, mas seus metadados (compostos pelo LOM e enriquecidos com outros, da própria API). Este componente consulta, periodicamente, os serviços nele registrados para garantir que seus metadados estejam atualizados, além de informações acerca da disponibilidade dos mesmos. 
O macroprocesso é um componente que visa orientar educadores quanto às fases a serem cumpridas no planejamento de trabalhos. É composto de 3 processos principais: concepção, definição de parâmetros e seleção de serviços. No primeiro, são definidos os parâmetros básicos (escopo, tema, etc.) do trabalho. O segundo refere-se à definição dos seus parâmetros específicos (relativo aos domínios de aprendizagem, avaliação, etc.). O último corresponde à seleção de serviços educacionais de modo a auxiliar o processo de planejamento ou mesmo a execução do trabalho. Cada processo engloba um conjunto de subprocessos voltados ao planejamento. $\mathrm{O}$ workflow de planejamento é definido como a seguir. Inicialmente, o docente, conforme suas necessidades de ensino, (a) especifica os parâmetros do trabalho, definidos nos metamodelos. Cada um o auxilia na especificação de um domínio específico (aprendizagem, avaliação, etc.). Logo, para cada domínio, (b) gera-se uma instância/modelo do metamodelo correspondente. Esse processo é chamado de instanciação, e cada instância engloba as variáveis que caracterizam uma conjuntura particular do domínio. É facultado ao educador (c) selecionar serviços educacionais para auxiliá-lo na instanciação dos modelos (por exemplo, gerando informações para tomada de decisão) ou na execução destes (exemplificado na seção 4.3).

Os serviços são disponibilizados em função dos parâmetros selecionados (desde que haja serviços que atendam à seleção). Para a criação de serviços compatíveis com o framework (e o ecossistema), faz-se necessário manter compatibilidade com a API e o metamodelo de planejamento. Assim, se no metamodelo de grupos, por exemplo, forem estabelecidos parâmetros que possibilitem a formação de grupos apenas por alunos, não poderão ser integrados à estrutura de ecossistema serviços que implementem estratégias cujos educadores sejam os responsáveis pelo processo. Os parâmetros dos metamodelos definem a "interface" para criação dos serviços. A (d) especificação do projeto/trabalho refere-se ao conjunto de modelos instanciados no processo de planejamento. O resultado do planejamento, portanto, é o conjunto de modelos que exprimem requisitos, restrições e objetivos de um contexto específico de ensino/aprendizagem.

\subsection{Cenário de Uso}

Nesta seção, apresenta-se um cenário de uso [Wholin, 2012] visando explicitar a viabilidade da infraestrutura proposta quanto ao planejamento de trabalhos em grupo e à integração de serviços educacionais à plataforma de ecossistema - estruturada com base em um AVA. O AVA usado no cenário foi o Moodle. Professor e aluno foram os atores. O foco do cenário é a etapa de formação dos grupos. Portanto, etapas anteriores não são discutidas e encerra-se o processo após a mesma. Contudo, a princípio, foram definidos os módulos do trabalho (programação, banco de dados, redes) e o número de alunos por grupo. A integração da estrutura de ecossistema ao Moodle foi executada, mas não será apresentada. O processo tem inicio com o educador acessando o Moodle e o curso C (no qual o trabalho é planejado). Realizadas as etapas iniciais para definição dos parâmetros básicos do trabalho, chega-se àquela voltada à formação de grupos. Nesta, o docente (a) seleciona os parâmetros obrigatórios/opcionais com base em um modelo de features que explicita conceitos definidos nos metamodelos. Para o cenário, conceitos relacionados à formação de grupo (metamodelo de grupo). Durante o processo, é facultado ao educador (b) pesquisar e selecionar serviços para auxiliá-lo na tomada de decisão ou execução do modelo de grupo sendo instanciado. Os parâmetros selecionados no processo são usados pelo ecossistema para filtrar os serviços disponíveis. A pesquisa ocorre de modo similar a uma loja de aplicativos. O docente analisa a descrição dos serviços e verifica se algum 
VI Congresso Brasileiro de Informática na Educação (CBIE 2017)

Anais do XXVIII Simpósio Brasileiro de Informática na Educação (SBIE 2017)

atende aos seus interesses. Para a tomada de decisão, o professor seleciona o serviço e o configura para execução. Nesse caso, o planejamento fica em estado de espera até que o mesmo seja executado. Para a execução do modelo (ao fim do planejamento), o docente cria uma atividade no AVA, associa o serviço à mesma e o configura para ser executado. A depender do serviço (do seu objetivo/proposta) para tomada de decisão, pode se fazer necessário, igualmente, criar uma atividade no AVA para sua execução.

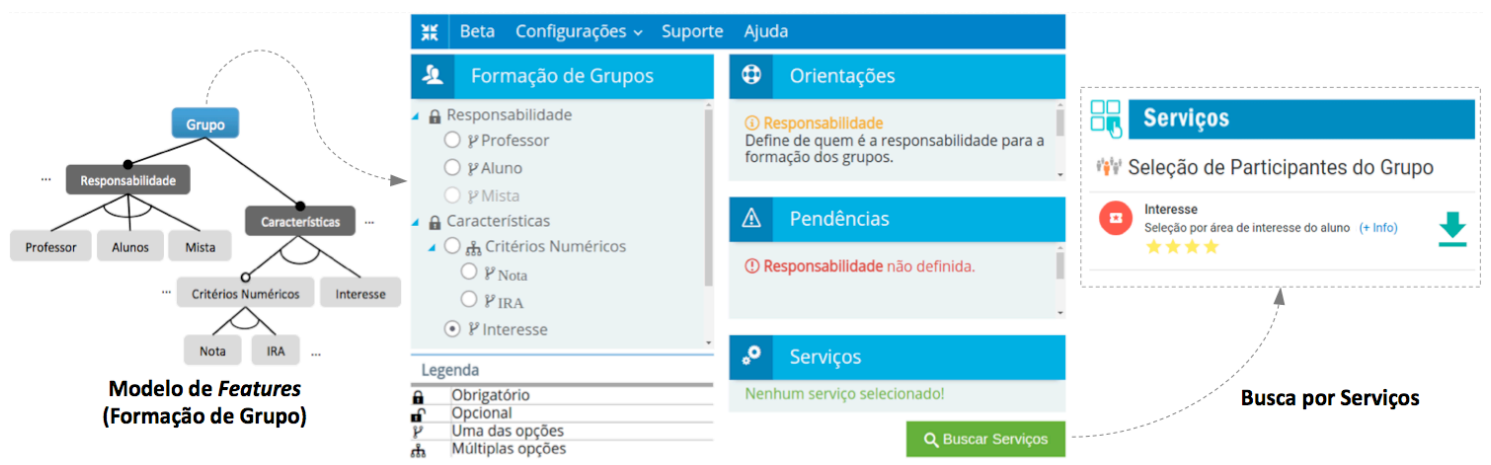

Figura 3. Interface de formação de grupos

Terminada a seleção, tem-se o modelo instanciado para formação de grupo - que expõe parâmetros para um contexto específico deste domínio. Com essa instanciação, o processo de planejamento (particularmente para este cenário) se encerra. O educador, de posse do trabalho/projeto estruturado, coloca-o em execução (configurando-o no AVA). Após o processo, o educador concede acesso aos alunos. O aluno acessa o AVA, o curso C, o trabalho e a tarefa de formação de grupos. Então, é redirecionado para uma página onde o serviço selecionado pelo educador é executado.

\subsection{Comparação com Propostas Relacionadas}

No contexto de planejamento de cenários de AC, uma tendência de pesquisa tem sido scripts colaborativos (SC). Um SC é um conjunto de instruções/diretrizes que possibilita estruturar e descrever cenários de AC, através da definição de componentes (atividades, alunos, recursos, etc.) e mecanismos (distribuição e sequenciamento de tarefas, formação de grupos, etc.) [Kobbe et al., 2007]. Seu objetivo é estimular e guiar a colaboração entre alunos [Dillenbourg, 2002]. Dada a complexidade intrínseca à sua estruturação, muitas ferramentas de autoria têm sido propostas para auxiliar educadores no processo [Challco et al., 2016; Alharbi et al., 2014; Fernández et al., 2013]. Embora algumas implementem modelos pedagógicos como modo de prover suporte educacional ao desenvolvimento de SC, nenhuma provê suporte ao PBL. Nessa perspectiva, a carência de recursos voltados ao mesmo [Massa et al., 2012] e as dificuldades ou desconhecimento dos educadores em relação às suas características [Martin et al., 2014] tornam complexa sua implementação. $\mathrm{O}$ framework proposto neste trabalho visa, portanto, diminuir essa carência - superando os desafios inerentes à sua implementação e, assim, tornando-o mais acessível.

Usualmente, a representação de scripts é realizada usando-se uma linguagem de modelagem educacional (EML) [Challco et al., 2016] - sendo IMS-LD a mais utilizada. $\mathrm{O}$ intuito é prover interoperabilidade, possibilitando que os scripts sejam executados em sistemas de gerenciamento de aprendizagem (LMS) compatíveis com a mesma. Apesar do suporte oferecido por tais ferramentas, o processo de planejamento é restrito aos seus recursos pedagógicos/técnicos; uma potencial limitação, dado o desafio das mesmas em acompanhar as constantes inovações do domínio educacional. A arquitetura proposta no 
presente trabalho, por outro lado, possibilita que recursos sejam providos por quaisquer desenvolvedores externos, dispensando-a de implementá-los - portanto, uma vantagem sobre as atuais abordagens. Em [Lorenzo et al., 2008], o uso de "recursos terceirizados" também é proposto. Contudo, trata-se de um LMS (não de uma ferramenta de autoria) logo, uma estrutura não voltada à criação de scripts, mas somente à sua execução. Além disso, scripts criados particularmente para esse LMS, visando usar recursos externos por ele providos, só podem ser executados no mesmo - um prejuízo à interoperabilidade. $\mathrm{O}$ framework apresentado nesta pesquisa oportuniza não apenas a autoria, mas a execução de cenários de aprendizagem. Ademais, o uso de recursos terceirizados não compromete a interoperabilidade, visto que a mesma é aplicada a toda estrutura de ecossistema, e não apenas ao arquivo de descrição do cenário (scripts), como se observa nas ferramentas de autoria existentes. Portanto, embora o trabalho/projeto especificado possa demandar, na sua execução, o uso de serviços externos, todo o processo está assentado na estrutura de ecossistema (que pode ser implementada sobre qualquer LMS).

\section{Conclusões}

Neste artigo, buscou-se analisar como professores, do ensino superior presencial em computação, planejam trabalhos em grupo em disciplinas de graduação. Realizou-se entrevistas com 30 professores de uma universidade federal. Os resultados evidenciaram que eles realizam o planejamento com base tão somente em suas próprias experiências e não definem parâmetros essenciais - principalmente com relação à orientação das ações dos alunos e a analise do aprendizado. A dificuldade dos docentes para com o processo indica a necessidade de prover-lhes orientação, expondo-os a parâmetros que devem ser aplicados. Neste sentido, propôs-se um framework para o planejamento de trabalhos em grupo baseados em PBL. O interesse por PBL está no fato do mesmo possibilitar tanto a aquisição de conhecimento quanto o desenvolvimento de relevantes habilidades sociais e profissionais. Assim, preparando alunos para situações que vivenciarão em ambientes reais de trabalho.

Quanto às limitações da pesquisa, aponta-se a não representatividade da amostra examinada. Entretanto, presume-se que os problemas identificados sejam intrínsecos ao contexto de trabalho em grupo e, portanto, verificados (mesmo que em parte) em outras amostras. É objetivo da pesquisa estender a análise para docentes de outras instituições (a princípio, mantendo-a no domínio da computação). Apesar do estudo exploratório ter fundamentado a proposição do framework, argumenta-se que a limitação não constituiu prejuízo à mesma - pois, o projeto arquitetural do framework lhe atribui adaptabilidade em termos de metamodelos (e, consequentemente, dos serviços a eles relacionados). De fato, não apenas estes podem ser estendidos (de modo a incorporar novas propriedades e parâmetros), como outros podem ser propostos e integrados ao framework. Os trabalhos futuros incluem, além da extensão da análise, concluir a estruturação dos metamodelos, finalizar a implementação do ferramental computacional e realizar um estudo de caso que envolva todo o processo de planejamento (todos os metamodelos).

\section{Referências}

Alharbi, N. M., Athauda, R. I., Chiong, R. (2014) A Survey of CSCL Script Tools That Suport Designing Collaborative Scenarios. Web and Open Access to Learning.

Barkley, E. F., Major, C. H., K., Cross, K. P. (2014). Collaborative Learning Techniques: A Handbook for College Faculty. San Francisco: Jossey-Bass. 
VI Congresso Brasileiro de Informática na Educação (CBIE 2017)

Anais do XXVIII Simpósio Brasileiro de Informática na Educação (SBIE 2017)

Challco, G. C., Bittencourt, I. Ig., Isotani, S. (2016). Computer-based systems for automating instructional design of collaborative learning scenarios: a systematic literature review. International Journal of Knowledge and Learning, 11(4).

Dillenbourg, P. (2002). Overscripting CSCL the risks of blending collaborative learning with instructional design. Three worlds of CSCL. Can we support CSCL? 61-91.

Fidalgo-Blanco, A., Sein-Echaluce, M. S., García-Peñalvo, F. J., Conde, M. A. (2015) Using Learning Analytics to improve teamwork assessment, C.H.B, 47, 149-156,

Fragoso, O. G., Santaolaya, R., Munoz, S. J., Valenzuela, B. D., Rojas, J. C. (2014). Integration of learning Web services into LMS. America/Panama Convention, 1-6.

Höver, K. M.; Mühlhäuser, M. (2014). Can We Use S-BPM for Modeling Collaboration Scripts?. Communications in Computer and Information Science. 174-187.

Isotani, S., Mizoguchi, R., Isotani, S., Capeli, O. M., Isotani, N., Albuquerque, A. R., Jaques, P. (2013). A Semantic Web-based authoring tool to facilitate the planning of CL scenarios compliant with learning theories. Computers/Education, 63, 267-284.

Isotani, S., Mizoguchi, R., Inaba, A., \& Ikeda, M. (2010). The foundations of a theoryaware authoring tool for CSCL design. Computers \& Education, 54(4), 809-834.

Kimble, C., Hildreth, P. M., \& Bourdon, I. (2008). Communities of practice: Creating learning environments for educators, Vol. 1. Charlotte, NC: Information Age Pub.

King, A. (2007). Scripting Collaborative Learning Processes: A Cognitive Perspective. Scripting Computer-Supported Collaborative Learning, 13-37.

Kobbe, L., Weinberger, A., Dillenbourg, P., Harrer, A., Hamalainen, R., F, F. (2007). Specifying Computer-Supported Collaboration Scripts. Journal CSCL, 2, 211-224.

Lorenzo, B. M. L., Sánchez, E. G., Gorgojo, G. V., Dimitriadis, Y. A., Pérez, J. I. A, Abellán, I. M. (2008). Gridcole: A tailorable grid service based system that supports scripted collaborative learning, Computers \& Education, 51, 155-172.

Manikas, K., Hansen, K. (2013) Software Ecosystems: A Systematic Literature Review. Journal of Systems and Software, 86(5), 1294-1306.

Martin, J. G., Carlos, L. L., Jorge, E. P. M. (2014). Supporting the Design and Development of Project Based Learning Courses. IEEE Frontiers in Education.

Massa, N., Dischino, M., Donnelly, J., Hanes, F., \& DeLaura, J., (2012). Problem-based learning in a pre-service technology and engineering education course. A.S.E.E

Muller, G. (2013). A reference architecture primer, Buskerud University College, 1-21.

Santos, R; Engenharia e Gerenciamento de Ecossistemas de Software (2013). Tese Doutorado. Pós-graduação em Engenharia de Sistemas e Computação, UFRJ.

Fernández, E. V., Leo, D. H, Pérez, J. I. A., Dimitriadis, Y. (2013). WebCollage: an implementation of support for assessment design in CSCL macro-scripts, 67, 79-97.

Weinberger, A., Kollar, I., Dimitriadis, Y., Mäkitalo-Siegl, K., \& Fischer, F. (2009). Computer-supported collaboration scripts. Educational psychology and CS, 155-173

Wholin, C., Runeson, P., Host, M., Ohlsson, M. Regnell, B., Wesslén, A. (2012) Experimentation in Software Engineering. Springer Berlin Heidelberg. 